\title{
Miocardiopatia Hipertrófica. Papel da Ecocardiografia Doppler no Diagnóstico e na Orientação Terapêutica
}

\author{
Airton José Hoss, Carlos Henrique Purper Petterson, Leonora Scherer \\ Santa Cruz do Sul, RS
}

A miocardiopatia hipertrófica (MH) caracteriza-se por apresentar hipertrofia simétrica ou assimétrica do miocárdio ventricular, geralmente com predomínio no septo interventricular(SIV), com ou sem obstrução dinâmica de via de saída ${ }^{1-3}$. A cavidade ventricular costuma ser de tamanho normal ou reduzida e apresenta disfunção diastólica resultante, principalmente, de déficit do relaxamento ventricular, mas também devido ao aumento da rigidez intrínseca da parede e da redução da câmara ventricular ${ }^{4,5}$. A função contrátil costuma ser normal ou hiperdinâmica. A MH pode se apresentar desde uma forma assintomática até situações de insuficiência cardíaca avançada ou de morte súbita, muitas vezes, sem nenhuma sintomatologia prévia, tornando o seu diagnóstico precoce difícil, porém, imprescindível em alguns casos ${ }^{6}$.

A ecocardiografia é o exame mais empregado atualmente na avaliação da $\mathrm{MH}$. Tem sido empregada tanto na investigação de casos suspeitos quanto para screening de parentes de pacientes com a doença. Através da ecocardiografia é possível avaliar os aspectos morfológicos, funcionais e hemodinâmicos da $\mathrm{MH}^{7}$.

A participação do ecocardiograma no estudo das miocardiopatias (MC) é cada vez mais decisiva em sua caracterização e no esclarecimento dos mecanismos fisiopatológicos envolvidos, com importantes implicações terapêuticas e prognósticas sendo, atualmente, o método de maior aplicabilidade nas várias formas de MC. Apesar do ecocardiograma ter alta sensibilidade para detectar a MC, tal constatação não é, em muitos casos, específica para este diagnóstico. Na prática, o diagnóstico de MC é realizado após a exclusão de todas as demais doenças cardiovasculares, que podem provocar dano miocárdico secundário, contudo, deve ser lembrado que essas doenças podem coexistir com uma MC.

\section{Aspectos ecocardiográficos}

Critérios diagnósticos - $\mathrm{O}$ achado ecocardiográfico característico na MH é a hipertrofia do ventrículo esquerdo

CENTROCOR - Centro de Diagnóstico Cardiovascular - Santa Cruz do Sul-RS Correspondência: Airton José Hoss - Rua Marechal Deodoro, 94 - 96810-110 Santa Cruz do Sul, RS

Recebido para publicação em 4/9/97

Aceito em 20/1/98
(VE), porém, há uma grande variabilidade no seu grau e padrão. A maioria dos pacientes (90\%) apresenta a forma assimétrica clássica com predomínio no $\operatorname{SIV}^{8}$ (tab. I). As demais paredes do VE costumam apresentar graus variáveis de hipertrofia, exceto a parede posterior (PP) que costuma ter espessura normal ${ }^{9}$. Nesta forma, o SIV geralmente apresenta uma espessura $\geq 15 \mathrm{~mm}$ (normal $\leq 10-11 \mathrm{~mm}$ ) e a relação SIV/PP é >1,3, fato pelo qual a MH é, algumas vezes, indevidamente chamada de hipertrofia septal assimétrica (HSA) ${ }^{10,11}$.

Mesmo porções contíguas do VE podem apresentar diferentes espessuras e a transição entre elas é, freqüentemente, abrupta. Dessa forma, em adultos, o diagnóstico de MH pode ser feito a partir da espessura da parede do VE, quando esta for $>13 \mathrm{~mm}^{12}$. Espessura de parede entre $11 \mathrm{e}$ $13 \mathrm{~mm}$ com ou sem relação SIV/PP $>1,3$ e sem causa aparente pode corresponder à forma incipiente de $\mathrm{MH}$, particularmente, quando há disfunção diastólica associada ${ }^{13,14}$. Recentemente, observaram-se achados anatomopatológicos de $\mathrm{MH}$ em pacientes ainda sem hipertrofia (forma préhipertrófica) ${ }^{15}$.

Distribuição da hipertrofia - Recentemente, Klues e $\mathrm{col}^{15}$ estudaram, ecocardiograficamente, 600 pacientes com MH. Nesse estudo, a espessura de parede variou de 15 a $52 \mathrm{~mm}$ (média 22), sendo >30mm em $11 \%$ e <20 mmem $45 \%$. Em relação à distribuição da hipertrofia, o SIV anterior esteve hipertrofiado em $96 \%$ dos pacientes; menos comumente esteve envolvido o SIV posterior (66\%), a parede lateral livre do VE (42\%) e a parede posterior livre do VE (18\%). Além disto, em $83 \%$ dos pacientes, $\mathrm{o}$ SIV anterior foi a região predominante da hipertrofia, seguido do SIV posterior (10\%), parede lateral (4\%) e parede posterior (2\%). Em $28 \%$

\begin{tabular}{|c|}
\hline \multicolumn{1}{|c|}{ Quadro I - Causas de MH } \\
\hline A - MH Idiopática \\
B - Condições Associadas com MH \\
Endocrinopatias \\
Acromegalia, hipotireoidismo, feocromocitoma. \\
Erros inatos do metabolismo \\
Glicogenose (doença de Pompe), doença de Fabry. \\
Doenças heredo-familiares, neurológicas e doenças neuro- \\
+musculares: \\
Ataxia de Friedreich, neurofibromatose, doença de Noonan, \\
lentiginose (síndrome de Leopard).
\end{tabular}




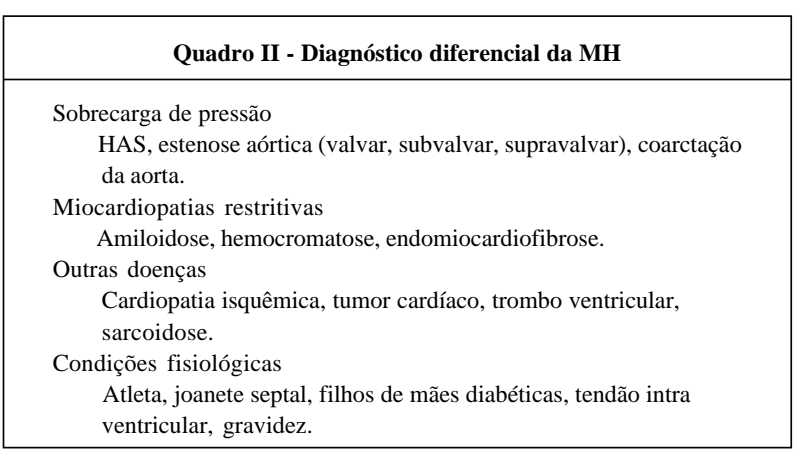

dos casos, o espessamento da parede foi confinado a um único segmento do ventrículo; em $38 \%$ envolveu dois segmentos (mais comumente o SIV anterior e posterior) e em $34 \%$ o envolvimento foi difuso, atingindo pelo menos três a quatro segmentos do VE. Alguma porção do SIV esteve envolvida no processo hipertrófico em $98 \%$ dos casos.

Houve uma relação direta entre o número de segmentos envolvidos e a espessura máxima da parede. Hipertrofia maciça $(>30 \mathrm{~mm})$ esteve presente em $23 \%$ dos pacientes com três ou mais segmentos envolvidos e, em apenas, $2 \%$ com um segmento. Quanto à distribuição no plano longitudinal, ahipertrofia foi proximal (basal)em 29\%, distal (apical)em $28 \%$ e em $36 \%$ distribuiu-se igualmente nas duas localizações.

Em 5\% dos pacientes com MH observou-se uma hipertrofia simétrica, envolvendo todas as paredes do VEna mesma proporção ${ }^{8}$. Eventualmente, o ventrículo direito(VD), sobretudo a via de saída, pode apresentar hipertrofia ${ }^{7,9}$.

A forma apical de $\mathrm{MH}$ caracteriza-se por espessamento miocárdico, somente, na ponta do ventrículo (em $99 \%$ dos casos o VE), ocorrendo em menos de $10 \%$ dos casos no nosso meio, porém, atingindo $25 \%$ dos pacientes com MH no Japão ${ }^{16}$.

Obstruçãoaofluxodesaída do VEemovimentoanterior sistólico da valva mitral - Cerca de $25 \%$ dos pacientes apresentam obstrução dinâmica da via de saída do ventrículo esquerdo (VSVE). A obstrução geralmente se dá, anteriormente, por hipertrofia do SIV na altura da via de saída do VEe, posteriormente, pela cúspide anterior da valva mitral. Um número variável de pacientes pode também apresentar obstrução, somente, após manobras provocativas (manobra de Valsalva, drogas vasodilatadoras, como nitratos, drogas estimulantes da contratilidade, como isoproterenol) ${ }^{8}$.

As cúspides da valva mitral estão anormalmente alongadas, alterando a geometria da VSVE e criando um gradiente de pressão ${ }^{15-19}$. Nesta situação, observam-se, via de regra, um movimento anterior sistólico (MAS) anormal da

\begin{tabular}{|lc|}
\hline \multicolumn{2}{|c|}{ Tabela I - Formas de miocardiopatia hipertrófica ${ }^{16}$} \\
\hline Forma & Frequiência (\%) \\
\hline Septal & 86 \\
Apical & 8,3 \\
Concêntrica & 3,2 \\
Outras & 2,5 \\
\hline
\end{tabular}

\begin{tabular}{|llll|}
\hline \multicolumn{4}{|c|}{ Tabela II - Fatores de confusão no diagnóstico da MH } \\
\hline Condição & HSA & OVSVE & MAS \\
\hline Miocardiopatia hipertrófica & $96 \%$ & $25 \%$ & $\pm 30 \%$ \\
Causas: & & & \\
Acromegalia & Possível & Possível & Possível \\
Hipotireoidismo & Possível & Possível & Possível \\
Feocromocitoma & Possível & Possível & Possível \\
Doença de Pompe & Possível & Possível & Não \\
Ataxia de Friedrich & Possível & Geralmente não & Não \\
Doença de Noonan & Possível & Geralmente não & Não \\
Diagnóstico diferencial & & & \\
HAS & $15 \%$ & Possível & Eventual \\
Amiloidose & Possível & Possível & Possível \\
Endomiocardiofibrose & Possível & Não & Não \\
Infarto do miocárdio & Possível & Não & Não \\
Massas cardíacas & Possível & Possível & Não \\
Atleta & Freqüente & Não & Não \\
Joanete septal & Não & Eventualmente & Não \\
Filhos de diabéticas & Possível & Não & Possível \\
Gestação & Possível & Possível & Não \\
\hline HSA- hipertrofia septal assimétrica; OVSVE- obstrução na via de saída do \\
ventrículo esquerdo; MAS- movimento anterior sistólico da valva mitral; HAS- \\
hipertensão arterial sistêmica. & & \\
\hline
\end{tabular}

cúspide anterior e, ocasionalmente, um posterior da valva mitral. Este MAS pode ser leve (a cúspide aproxima-se do septo, mas não há contato), moderado (leve contato septal) ou severo (prolongado contato septal, mais de $30 \%$ da sístole ecocardiográfica). Klues e $\mathrm{col}^{20}$ demonstraram que acentuado MAS foi mais comum em pacientes com hipertrofia extensa e difusa (38\%), que em pacientes com apenas um segmento envolvido (25\%) e naqueles com hipertrofia, predominantemente proximal (36\%), que com distal (22\%). Além disso, esses pacientes tinham maiores espessuras de parede que aqueles com MAS leve.

Mesmo na MH simétrica, pode ocorrer obstrução dinâmica da VSVE ${ }^{5}$. Em alguns pacientes ocorre aumento predominante na porção média do SIV, podendo causar obstrução médio-ventricular. Quando o VD está envolvido, pode haver obstrução de via de saída do VD (VSVD) ${ }^{5,21}$.

Insuficiência mitral - A MH, freqüentemente, leva à regurgitação mitral (40a 75\%), tanto em pacientes com a forma obstrutiva quanto naqueles sem obstrução na VSVE ${ }^{22-25}$. Esta regurgitação geralmente aparece na sístole tardia, principalmente, nos casos obstrutivos. Oki e col ${ }^{26}$ observaram uma freqüência de mais de $90 \%$ de regurgitação mitral com ecocardiografia transesofágica e de $44 \%$ de prolapso de valva mitral (PVM). A regurgitação mitral nesses pacientes pode ser explicada por uma insuficiente coaptação das cúspides da valva mitral associada com anormalidades funcionais dos músculos papilares, gerando uma desproporção espacial entre o VE estreitado e o aparato mitral. Os músculos papilares então protuem para dentro da cavidade ventricular, causando redundância da cordoalha tendínea, o que pode gerar o prolapso da cúspide anterior (31\%) e posterior (13\%).

Alguns autores, entretanto, não consideram o PVM como uma conseqüência da $\mathrm{MH}$, mas sim como parte de uma 
desordem genética, que associa as duas condições ${ }^{27}$. Também, é de se esperar que em pacientes com obstrução na VSVE, a regurgitação mitral ocorra durante ou após o MAS ${ }^{28,29}$; entretanto, a regurgitação mitral já fora observada antes do MAS, nesses casos sendo, possivelmente, causada por uma estimulação mecânica resultante da exposição da cúspide a fluxos de alta velocidade na saída do VE estreitado e de fatores congênitos ${ }^{26}$.

MAS e gradiente ventricular esquerdo não são patognomônicos de $\mathrm{MH}$, podendo ocorrer em outros estados hipercontráteis, hipertrofia ventricular esquerda de diversas outras etiologias, transposição de grandes vasos e infiltração do septo ${ }^{30}$.

Cavidade ventricular esquerda - A cavidade ventricular esquerda na MH está, via de regra, normal ou diminuída. A dimensão diastólica da cavidade do VEé $<45 \mathrm{~mm}$ na maioria dos pacientes com $\mathrm{MH}$, podendo se tornar $>55 \mathrm{~mm}$ nos estágios finais, nos quais disfunção sistólica é associada $^{5,31}$. Geralmente, a função contrátil global está normal ou hiperdinâmica. Quase sempre se detecta algum grau de disfunção diastólica, geralmente, com padrão de relaxamento alterado (disfunção diastólica inicial). As formas mais graves podem apresentar padrão pseudo-normal ou até restritivo ${ }^{5}$. As anormalidades da função diastólica podem ser demonstradas pela ecocardiografia e Doppler em $80 \%$ dos pacientes. Comumente, ocorre aumento do átrio esquerdo secundário à disfunção diastólica do VE e/ou à insuficiência mitral ${ }^{5}$.

Outros achados - Outros achados ecocardiográficos podem ainda estar presentes em pacientes com $\mathrm{MH}$, como diminuição da mobilidade do septo e mobilidade normal ou aumentada da parede posterior. Um padrão granular pode ser visto nas porções hipertrofiadas do miocárdio em alguns pacientes com $\mathrm{MH}$, podendo ser usado para distinguir a condição das miocardiopatias secundárias ${ }^{32}$.

\section{Condições associadas com miocardiopatia hipertrófica.Aspectos ecocardiográficos peculiares}

A MH idiopática responde por quase a totalidade dos casos, contudo, algumas doenças podem causar $\mathrm{MH}$, comumente simétrica (quadro I). Em algumas destas, eventualmente, observa-se HSA e/ou MAS, com ou sem obstrução dinâmica da VSVE. A acromegalia, o hipotireoidismoe o feocromocitoma podem apresentar HSA, inclusive com MAS e obstrução da VSVE ${ }^{13,33}$. No hipotireoidismo podese também observar hipocinesia do SIV e derrame pericárdico ${ }^{13}$. A doença de Pompe pode apresentar HSA e MAS, porém, costuma apresentar disfunção ventricular sistólica associada ${ }^{13,34}$. Na doença de Pompe e na doença de Fabry, observa-se ecodensidade granular das paredes ventriculares ${ }^{35}$. A ataxia de Friedreich e a doença de Noonan podem apresentar HSA, geralmente sem obstrução da VSVE ${ }^{13,36}$.

\section{Diagnóstico diferencial}

Inúmeras condições podem ser confundidas com $\mathrm{MH}$ (tab. II) e, em algumas ocasiões, o diagnóstico diferencial pode ser particularmente difícil, pois muitas vezes, tais condições podem coexistir (quadro II). Em casos duvidosos, a avaliação por ecocardiograma dos familiares diretos pode denunciar o caráter familiar da afecção, o que é encontrado na MHidiopática ${ }^{37}$.

Hipertrofia secundária à sobrecarga de pressão - $\mathrm{Na}$ hipertensão arterial sistêmica (HAS), a hipertrofia ventricular geralmente é simétrica, porém, cerca de $15 \%$ dos pacientes apresentam HSA ${ }^{13}$, eventualmente, com MAS mitral, com ou sem obstrução da VSVE ${ }^{5,38}$ - lembrar que HSA nãoé sinônimo de MH. Grau leve de hipertrofia ventricular esquerda (10 a 13mm) restrita ao SIV com ou sem relação SIV/ $\mathrm{PP}>1,3$ é, freqüentemente, encontrada na $\mathrm{HAS}^{5,13}$. O mesmo aspecto de hipertrofia pode ser encontrado na estenose aórtica e na coarctação da aorta ${ }^{5}$. Qualquer doença que curse com hipertensão arterial pulmonar, como a estenose mitral, pode causar hipertrofia do VD e do SIV, com achado ecocardiográfico de $\mathrm{HSA}^{5,13}$.

Miocardiopatias restritivas - A amiloidose e a hemocromatose podem ser confundidas com $\mathrm{MH}$, principalmente em seus estágios mais precoces ${ }^{3,39}$. A amiloidose pode, inclusive, apresentar HSA com MAS mitral e obstrução na VSVE $^{35}$. A ecodensidade granular difusa das paredes ventriculares, o envolvimento de todas as paredes cardíacas, inclusive, com espessamento do septo interatrial, e alterações nas válvulas cardíacas são dados que favorecem este diagnóstico ${ }^{39}$. A endomiocardiofibrose com envolvimento apical do VE pode simular $\mathrm{MH}$ apical. A fibroelastose endocárdica (forma restritiva) também pode produzir quadro semelhante à MH simétrica em crianças.

Outras doenças - Infarto do miocárdio ínfero-posterior pode apresentar relação $S I V / P P>1,3$ devido à diminuição da espessura da parede com necrose e/ou hipertrofia compensatória do SIV 13,36. Tumores cardíacos e granulomas sarcóides podem causar espessamento assimétrico da parede ventricular $^{13,33}$. Trombo ventricular mural, principalmente em região apical, não deve ser confundido com MH apical.

Condições fisiológicas - No atleta, pode-se observar hipertrofia ventricular esquerda e, embora a região predominante da hipertrofia sempre envolva o SIV anterior, geralmente, outros segmentos da parede são igualmente envolvidos. Além disso, a cavidade ventricular esquerda no final da diástole é, geralmente, $>55 \mathrm{~mm}$ em mais de um terço dos atletas altamente treinados ${ }^{7}$. O septo entre 13 a $16 \mathrm{~mm}$ não distingue o chamado coração de atleta da MH, sendo necessário, nesses casos, controle ecocardiográfico seriado desses pacientes. Espessura septal acima de 16mm é fortemente sugestivo de $\mathrm{MH}^{40}$. A função diastólica normal favorece o diagnóstico de hipertrofia fisiológica ${ }^{41}$. 
Em idosos, ocorre aumento da angulação entre o SIV e a aorta, o que se relaciona a um espessamento e projeção do SIV basal para a VSVE (SIV sigmóide ou joanete septal), podendo causar fluxo turbulento e, eventualmente, obstrução na VSVE ${ }^{34,38}$. Filhos de mãe diabética podem apresentar HSA com ou sem MAS mitral ${ }^{30,32}$. Tendões fibrosos no VE podem causar pequeno espessamento da parede ao nível de suas inserções e, às vezes, situam-se paralelo ao SIV, podendo causar uma falsa imagem de $\mathrm{HSA}^{39}$. Efeito similar pode ser provocado pela banda moderadora do VD. Recentemente foi descrita hipertrofia ventricular esquerda com HSA e obstrução da VSVE secundário à gravidez ${ }^{42}$.

\section{Implicações clínico-terapêuticas}

Não há evidências definitivas na literatura que sustentem um aumento na sobrevida dos pacientes com $\mathrm{MH}$, utilizando-se qualquer tipo de tratamento clínico. Os sintomas de insuficiência cardíaca estão mais relacionados ao grau de disfunção diastólica do que à extensão da hipertrofia ou a obstrução da VSVE ${ }^{5,8}$. Geralmente, há uma correlação entre o grau de HVE e a disfunção diastólica ${ }^{5}$. Os betabloqueadores aliviam os sintomas e melhoram a tolerância ao exercício por diminuírem a frequiência cardíaca e melhorarem o enchimento diastólico e, também, por diminuírem o gradiente da VSVEno exercício. O verapamil também melhora o enchimento ventricular e, provavelmente, diminui a isquemia miocárdica nesses pacientes. $\mathrm{O}$ digital deve ser reservado para os casos onde haja disfunção contrátil associada ou para o controle da freqüência cardíaca na fibrilação atrial, pois a taquicardia pode induzir ou agravar a obstrução da VSVE ${ }^{4}$. Os vasodilatadores arteriais devem ser evitados, pois podem causar taquicardia reflexa com diminuição do enchimento ventricular, além de agravar o gradiente de VSVE na MH obstrutiva ${ }^{4}$.

A resposta ao tratamento pode ser avaliada pela ecocardiografia através da observação do padrão de enchimento ventricular, tempo de relaxamento isovolumétrico, gradiente da VSVE, hipertensão arterial pulmonar, etc ${ }^{4,13,33}$.

Nos pacientes em que o tratamento farmacológico falhou em controlar os sintomas, pode-se optar por colocação de marcapasso de dupla câmara, cujo papel no alívio dos sintomas é incerto, mas parece diminuir o gradiente na VSVE nos pacientes com obstrução da mesma e aumentar o débito cardíaco. A cirurgia de miotomia-miectomia para pacientes com obstrução da VSVE é outra opção terapêutica, e há algumas evidências indicando que este tipo de terapia poderia melhorar a sobrevida de pacientes com MH e obstrução naVSVE.

O ecocardiograma pode ainda ser útil na seleção de pacientes para o tratamento cirúrgico, visto que, para a realização de miomectomia, é necessário que haja obstrução significativa de VSVE, VSVD ou médio-ventricular. Quando há regurgitação mitral acentuada associada, pode ser necessária a troca valvar mitral ${ }^{5,43,44}$. Aos pacientes sem estas características, resta apenas o transplante cardíaco, caso a terapêutica cirúrgica seja indicada ${ }^{45}$. $\mathrm{O}$ ecocardiograma transesofágico transoperatório pode auxiliar no planejamento da extensão da miomectomia, avaliar resultados imediatos, como o gradiente da VSVE e o grau de regurgitação mitral, bem como excluir complicações ${ }^{46}$.

Nos pacientes que já evoluíram para dilatação e disfunção sistólica, deve-se realizar o tratamento tradicional para insuficiência cardíaca (inibidores da enzima de conversão, digital, diuréticos, outros vasodilatadores).

\section{Aspectos evolutivos}

A MH idiopática responde pela quase totalidade dos casos. Mais de $50 \%$ têm caráter familiar e costuma ser detectada somente na adolescência ou na idade adulta. Após a idade adulta, geralmente, não ocorre significativa progressão da hipertrofia ${ }^{5}$. Mais da metade dos pacientes permanece estável durante o acompanhamento; outros apresentam piora do quadro principalmente devido à progressiva obstrução da VSVE, muitas vezes associada à insuficiência mitral ${ }^{47,48}$. Nos estágios finais pode ocorrer diminuição da hipertrofia e dilatação ventricular com disfunção sistólica ${ }^{5}$. A história natural da doença parece indicar uma mortalidade de cerca de $3 \%$ ao ano ${ }^{30}$.

A avaliação ecocardiográfica de todos os familiares diretos de um paciente com MH torna-se mandatória, devido ao risco de morte súbita associado a esta condição. Mesmo na forma "pré-hipertrófica" ocorre um risco aumentado de morte súbita ${ }^{9,14,48}$.

$\mathrm{Na}$ presença de hipertrofia ventricular esquerda leve (geralmente do SIV) sem causa aparente, deve-se suspeitar de MH incipiente e fazer acompanhamento ecocardiográfico seriado. Alteração da função diastólica (padrão de relaxamento alterado) pode ser sinal precoce de $\mathrm{MH}$ mesmo na ausência de hipertrofia ${ }^{14}$.

Algumas condições associadas com $\mathrm{MH}$ requerem tratamento específico, podendo inclusive levar à regressão do quadro e devem ser suspeitadas, principalmente, em pacientes com alterações músculo-esqueléticas, fácies sindrômico ou com endocrinopatia.

O ecocardiograma pode ainda ajudar na estratificação de risco para morte súbita, podendo identificar pacientes com pior prognóstico: parede ventricular $>20 \mathrm{~mm}$, gradiente sistólico máximo ao VSVE >50mmHg, disfunção diastólica padrão restritivo, disfunção sistólica associada e grande extensão da hipertrofia ${ }^{5,49,50}$.

$\mathrm{Na} \mathrm{MH}$, o exercício físico pode desencadear morte súbita. Pacientes com espessura da parede ventricular $>20 \mathrm{~mm}$ ou gradiente sistólico máximo VSVE $>50 \mathrm{mmHg}$, são considerados de "alto risco", não devem praticar esporte competitivo e têm contra-indicação para realizar prova ergométrica ${ }^{50,51}$. Os pacientes com MH obstrutiva têm contra-indicação relativa para realizar anestesia tipo bloqueio epidural, a qual deve ser evitada, particularmente, no grupo de alto risco citado ${ }^{5,52}$.

\section{Comentários finais}

A ecocardiografia Doppler tem um papel indispensá- 
vel no diagnóstico e na descrição dos diversos tipos morfológicos de MH e na definição dos mecanismos de obstrução da VSVE, regurgitação mitral e outras condições associadas. É de fundamental importância no screening dos familiares diretos de um paciente com MH e na detecção de casos de pior prognóstico, ajudando o clínico a orientar sua conduta terapêutica. Tem especial valor, ainda, na avaliação da resposta ao tratamento e no controle da evolução dos casos diagnosticados. Entretanto, cabe ressaltar que o ecocardiograma na $\mathrm{MH}$, assim como em outras doenças, descreve os achados do momento do exame, os quais podem apresentar significativa alteração, espontaneamente, ou devido ao tratamento, motivo pelo qual o clínico não deve considerar os resultados de um único exame definitivos.

\section{Referências}

1. Abelmann WH - Classification and natural history of primary myocardial disease. Prog Cardiovasc Dis 1984; 27: 73-94.

2. Wenger NK, Goodwin JF, Roberts WC - Cardiomyopathy and myocardial involvement in systemic heart disease. In: Hurst JW, ed. New York: McGraw-Hill, 1986: 1181 .

3. Keren A, Popp R - Assignment of patients into the classification of cardiomyopathies. Circulation, 1992; 86: 1622-33.

4. Stauffer JC, Gaasch WH-Recongnition and treatment of left ventricular diastolic dysfunction. Prog cardiovas Dis 1990; 32: 319-32.

5. Zipes DP, Rowlands DJ - Hipertrophic cardiomyopathy. In: Progress Cardiology: Philadelphia: Lea and Febiger, 1989

6. Spirito P, Seidman CE, Mc Kenna WJ et al - The management of hypertrophic cardiomyopathy. N Engl J Med 1997, 336: 775-85.

7. Maron BJ, Gardin JM, Flack JMet al-Prevalence of hypertrophic cardiomyopathy in a general population of young adults. Echocardiographic analysis of 4111 subjects in the CARDIA Study. Circulation 1995; 92: 785-9.

8. Wigle ED, Sasson Z, Henderson MA et al - Hypertrophic cardiomyopathy - The importance of the site and the extense of hypertrophy: a review. Prog Cardiovasc Dis 1985; 28: 1-83.

9. Maron BJ - Right ventricular cardiomyopathy. Another cause of sudden death in young. N Engl J Med 1988; 318: 178-80.

10. Bhandari AK, Nandsa NC - Myocardial texture characterization bu two-dimensional echocardiography. Am J Cardiol 1983; 51: 817-26.

11. Haertel JC - Abordagem ecodopplercardiográfica da miocardiopatia hipertrófica. Rev Bras Ecocardiografia 1989; 2: 3-23.

12. Pelliccia A, Maron BJ, Spataro A et al - The upper limit of physiologic cardiac hypertrophy in the highly trained elite athletes. N Engl J Med 1991; 324: 295 301.

13. Hagan AD, Di Sessa TG, Bloor CM et al - Two-dimensional echocardiography Clinical-pathological correlations in adult and congenital hearth disease. Boston: Little, Brown, 1983.

14. Mc Kenna WJ, Stewart TJ, Nihoyannopoulos SP et al - Hypertrophic cardiomyopathy without hypertophy: two families with myocardial disarray in the absence of increased myocardial mass. Br Hearth J 1990; 63: 287-90.

15. Klues HG, Roberts WC, Maron BJ - Morphological determinants of echocardiographic patterns of mitral valve systolic anterior motion in obstrutive hypertrophic cardiomyopathy. Circulation 1993; 87: 1570.

16. Albanesi $\mathrm{F}^{\circ} \mathrm{FM}$, Castier MB, Diamant JDA, Lopes JS, Lopes AS, Ginefra P Cardiomiopatia hipertrófica apical acometendo o ventrículo direito. Arq Bras Cardiol 1997; 68: 119-124.

17. Mautner SL, Klues HG, Mautner GC et al - Comparison od mitral valve dimensions in adults with valvular aortic stenosis, pure aortic regurgitation and hypertrophic cardiomyopathy. Am J Cardiol 1993; 71: 949.

18. Klues HG, Maron BJ, Dollar AL et al - Diversity of structural mitral valve alterations in hypertrophic cardiomyopathy. Circulation 1992; 85: 1651.

19. Grigg LE, Wigle ED, Williams WG et al - Transesophageal Doppler echocardiography in obstructive cardiomyopathy: classification of pathophysiology and importance in intraoperative decision making. J Am Coll Cardiol 1992; 20: 42 .

20. Klues HG, Schiffers A, Maron BJ - Phenotypic spectrum and patterns of left ventricular hypertrophy in hypertrophic cardiomyopathy: morphologic observations and significance as assessed by two-dimensional echocardiography in 600 patients. J Am Coll Cardiol 1995; 26: 1699-708.

21. Saxon LA, Stevenson WG, Middlekauff HR et al - Predicting death from progressive heart failure secondary ischemic or idiopathic dilated cardiomyopathy. Am J Cardiol 1993; 72: 62-5.

22. Cohen J, Effat H, Goodwin JF et al - Hypertrophic obstructive cardiomyopathy. Br Hearth J 1964; 26: 16-32.

23. Simon AL, Ron J, Gault J - Angiographic anatomy of the left ventricle and mitral valve in idiopathic hypertrophic subaortic stenosis. Circulation 1967; 36: 852-67.
24. Kinoshita N, Nimura Y, Ikamoto M et al -Mitral regurgitation in hypertrophic cardiomyopathy: non-invasive study by two-dimensional Doppler echocardiography. Br Heart J 1983; 49: 574-83.

25. Yonezawa Y, Nihoyannopoulas P, Mc Kenna WJ et al - Mitral regurgitation hypertrophic cardiomyopathy: a color doppler echocardiographic study. Am J Noninvas Cardiol 1988; 2: 195-8.

26. Oki T, Fukuda N, Iuchi A et al - Transesophageal echocardiographic evaluation of mitral regurgitation in hypertrophic cardiomyopathy: contributions of eccentric left ventricular hypertrophy and related abnormalities of the mitral complex. J Am Soc Echocardiography 1995; 8 : 503-10.

27. Rubenstein SR, Christodoulou JP, Arena FP et al - Coexisting hypertrophic hearth disease and mitral valve prolapse. Chest 1980; 78: 51-4

28. Gardin JM, Dabestani A, Glasgow GA et al - Echocardiographic and Doppler flow observations in obstructed and non obstructed hypertrophic cardiomyopathy. Am J Cardiol 1985; 56: 614-21.

29. Nishimura RA, Tajik AJ, Feeder GS et al - Evaluation of hypertrophic cardiomyopathy by color flow imaging: initial observations. Mayo Clin Proc 1986; 61: 631-9.

30. Braunwald E - Hearth disease. $5^{\text {th }}$ ed. Philadelphia: WB Saunders 1997: 1414 26.

31. Maron BJ, Pelliccia A, Spirito P-Cardiac disease in young trained atheletes. Insights into methods for distinguishing athlete's hearth from structural hearth disease, with particular emphasis on hypertrophic cardiomyopathy. Circulation 1995; 91: 1596-601.

32. Lattanzi F, Spirito P, Picasso E et al - Quantitative assessment of ultrassonic myocardial reflectivity in hypertrophic cardiomyopathy. J Am Coll Cardiol 1991; 17: 1085 .

33. Feigenbaum H - Echocardiography. $4^{\text {th }}$ edition. Philadelphia: Lea and Febiger 1986.

34. Zielinski P - Avaliação ecocardiográfica das miocardiopatias na infância. Rev Bras Ecocardiografia 1989; 2: 25-34.

35. Picano E, Pinamonti B, Ferdeghini EM et al - Two-dimensional echocardiography in myocardial amyloidosis. Echocardiography 1991; 8: 253-9.

36. Morcef FP - Ecocardiografia Uni-Bidimensional e Doppler. Rio de Janeiro: Revinter, 1990.

37. ACC/AHJ - Guidelines for the clinical application of echocardiography - A report of the ACC/AHJ task force on assessment of diagnostic on therapeutic cardiovascular procedures. Circulation 1990; 82: 2322-45.

38. Topol EJ, Traill TA, Fortuin NJ - Hipertensive hypertrophic cardiomyopathy in elderly. N Engl J Med 1985; 312: 277-83.

39. Klein AL, Tajik J - Doppler assessment of diastolic function in cardiac amyloidosis. Echocardiography 1991; 8: 233-51.

40. Maron BJ - Structural features of the athlete hearth as defined by echocardiography. J Am Coll Cardiol 1986; 7: 190-203.

41. Farlane $\mathrm{N}$ - A comparative study of left ventricular structure and function in elite athletes. Br J Sports Med 1991; 25: 45-8.

42. Rosenblatt JÁ, Osher HL, Lambrew CT - Transient hypertrophic obstructive cardiomyopathy during pregnany. Echocardiography 1994; 11: 105-10.

43. Shabetai R-Cardiomyopathy: How far have we come in 25 years, how far yet to go? J Am Coll Cardiol 1983; 1: 252-63.

44. Maron BJ, Mc Intosh CL, Klues HG et al - Morpologic basis for obstruction to right ventricular outflow in hypertrophic cardiomyopathy. Am J Cardiol 1993; 71: 1089-94.

45. Shirani J, Maron BJ, Cannon RO, Shahin S, Roberts WC-Clinicopathologic features of hypertrophic cardiomyopathy managed by cardic transplantation. Am J Cardiol 1993; 72: 434-40.

46. Marwick TH, Stewart WJ, Lever HM et al - Benefit of intraoperative echocardiography in the surgical management of hypertrophic cardiomyopathy. J Am Coll Cardiol 1992; 20: 1066-72.

47. Panza JÁ, Maris TJ, Maron BJ-Development and determinants of dynamic obstruc- 
tion to left ventricular outflow in young patients with hypertrophic cardiomyopathy. Circulation 1992; 85: 1398-405.

48. Hecht GM, Panza JÁ, Maron BJ - Clinical curse of middle-aged assyntomatic patients with hypertrophic cardiomyopathy. Am J Cardiol 1992; 69: 935-40.

49. Devereux RB - Left ventricular diastolic dysfunction: Early diastolic relaxation and late diastolic compliance. J Am Coll Cardiol 1989; 13: 337-9.

50. Bethesda conference report: cardiovascular abnormalities in the athlete: recom- mendations regarding eligibility for competition-task force III: hypertrophic cardiomyopathy, other myocardial diseases and mitral valve prolapse. J Am Coll Cardiol 1985; 6: 1215-17.

51. AHA Medical/Scientific Statement - Exercise standards: A statement for health professionals from the AHA. Circulation 1990; 82: 2286-322.

52. Goldman L-Evaluation of the cardiac patient for noncardiac surgery. ACC Current J Review 1994; 3: 60-3. 